\title{
30 days in medicine
}

\section{Increasing emergence of drug- resistant gonorihoea}

New data from 77 countries show that gonorrhoea is becoming increasingly resistant to treatment, and high-income countries in particular are reporting infections that are impossible to treat.

New data from the World Health Organization global gonococcal antimicrobial surveillance programme, which monitors trends in drug-resistant gonorrhea, were published in PloS Medicine. Nearly all countries (97\%) that report data found strains resistant to ciprofloxacin and $81 \%$ of countries reported increasing resistance to azithromycin. Even more worrying, $66 \%$ of countries reported the emergence of strains resistant to the current last-resort treatment, extended-spectrum cephalosporins (ESCs), oral cefixime or injectable ceftriaxone. In addition, three countries - Japan, France and Spain reported gonorrhoea superbugs.

Although most verified treatment failures are from high-income countries, this is probably a reflection of surveillance levels in resource-poor countries and may not reflect the true burden of ESC treatment failure.

Wi T, Lahra MM, Ndowa F, et al. Antimicrobial resistance in Neisseria gonorrhoeae: Global surveillance and a call for international collaborative action. PLoS Med 2017;358:e1002344. https://doi.org/10.1371/journal. pmed. 1002344

\section{Reduced radiotherapy effective in treatment of early breast cancer}

Radiotherapy targeted to the original tumour site or a lower dose to the whole breast is associated with similiar low rates of local tumour recurrence as standard radiotherapy to the whole breast and fewer longterm adverse effects in women treated for early breast cancer, according to a large randomised trial in the UK, published recently in The Lancet.

The study randomised 2016 women who had undergone breastconserving surgery for early breast cancer to three radiotherapy treatment groups: standard 40 Gy whole-breast radiotherapy, a reduced regimen of $36 \mathrm{~Gy}$ to the whole breast, and $40 \mathrm{~Gy}$ to the tumour site only. Radiotherapy was given in 15 daily fractions. After a median follow-up of just over 6 years, local relapse occurred in 9 patients $(1 \%)$ given whole breast radiotherapy, in $3(<1 \%)$ of those treated with reduced-dose radiotherapy and in $6(1 \%)$ of those given radiotherapy to the tumour site only.

The 5-year estimated cumulative incidence of local relapse was $1.1 \%$ (95\% confidence interval (CI) $0.5-2.3$ ) in the whole-breast radiotherapy group, $0.2 \%$ ( $95 \%$ CI $0.02-1.2 \%)$ in the reduceddose group, and $0.5 \%(95 \%$ CI $0.2-1.4 \%)$ in the partial-breast radiotherapy group.

Clinical, photographic and patient assessments showed similar rates of adverse effects after reduced-dose or partial-breast radiotherapy, with changes in breast appearance and breast hardness being significantly lower than with whole-breast radiotherapy.

Coles CE, Griffin CL, Kirby AM, et al. Partial breast radiotherapy after breast conservation surgery for patients with early breast cancer (UK IMPORT LOW trial): 5 year results from a multicentre, randomised, controlled, phase 3, non-inferiority trial. Lancet 2017 (epub 2 August 2017). https://doi.org/10.1016/S01406736(17)31145-5

\section{Labile blood pressure associated with higher risk of dementia}

Labile blood pressure may raise the risk of dementia, according to research published in Circulation. In the study, more than 1600 Japanese adults without dementia, with an average age of 71 years, were asked to measure their blood pressure at home for a month. More than half the study participants were women. Participants measured their blood pressure three times in the morning before eating breakfast or taking medication. Participants included normotensives and hypertensives, $40 \%$ of whom were taking medication for hypertension. Researchers reviewed the blood pressure readings, conducted cognitive testing to uncover early dementia, and reviewed records for occurrence of stroke.

In 5 years of follow-up, 134 participants developed Alzheimer's disease and 47 developed vascular dementia. Those with the highest variability in systolic blood pressure were more than twice as likely to develop any type of dementia or Alzheimer's disease, and nearly three times as likely to develop vascular dementia.

Oishi E, Ohara T, Sakata S, et al. Day-to-day blood pressure variability and risk of dementia in a general Japanese elderly population. Circulation 2017;136(6):516-525. https://doi.org/10.1161/ CIRCULATIONAHA.116.025667

\section{Disadvantaged children may have a higher risk of later heart disease}

Researchers from the Murdoch Children's Research Institute have found that children from socially and economically disadvantaged families and neighbourhoods seem to have thicker carotid artery walls, which in adults might suggest a higher risk for heart attack and stroke in later life. The study, published in the Journal of the American Heart Association, looked at family and neighbourhood socioeconomic data from 1477 Australian families and measured income, education, occupation of parents, and the relative socioeconomic status of the immediate neighbourhood.

They found that between the ages of 11 and 12 years, when the children's right carotid artery was measured for maximum carotid intima-media thickness, both family and neighbourhood socioeconomic position were associated with carotid artery inner-layer thickness, and that family association was the strongest relationship. Children whose families were in the bottom quartile, i.e. the most disadvantaged, were $46 \%$ more likely to have thicker measurements. Researchers postulated that infection and inflammation may be among the underlying factors, since infection is more common in more disadvantaged people.

Liu RS, Mensah FK, Carlin J, et al. Socioeconomic position is associated with carotid intima-media thickness in mid-childhood: The longitudinal study of Australian children. J Am Heart Assoc 2017;6:e005925. https:// doi.org/10.1161/JAHA.117.005925

\section{B Farham}

Editor

ugqirha@iafrica.com 